\title{
Domain growth and aggregation dynamics in Photo-induced phase transition phenomena
}

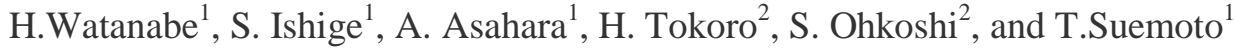 \\ ${ }^{1}$ The institute for solid state physics, the University of Tokyo \\ ${ }^{2}$ School of Science, the University of Tokyo \\ *hwata@issp.u-tokyo.ac.jp
}

\begin{abstract}
We have studied domain growth dynamics in photo-induced phase transition phenomena on $\mathrm{RbMn}\left[\mathrm{Fe}(\mathrm{CN})_{6}\right]$ by measuring the time developments of the Raman peaks of the $\mathrm{CN}$ stretching modes, which are sensitive to the boundaries between high temperature and low temperature domains. Under the high excitation intensity, we observe abrupt increase and slow decrease of the boundary. This indicates creation of the small domains and subsequent growth process with domain aggregation. Experimental results are explained qualitatively by the mean field theory with the pair valence states of $\mathrm{Fe}$ and Mn pairs taking into account of instability of domain boundary.
\end{abstract}

Keyword photo-induced phase transition phenomena, Raman spectroscopy, Domain dynamics

\section{Introduction}

Recently, photo-induced phase-transition (PIPT) phenomena have been studied in various systems and the dynamical processes of creating the photo-induced phases have been clarified in a wide time range $[1,2,3,4,5]$. Although the domain creation dynamics is an important issue and has been attracting much attention, relatively less information has been obtained. It is difficult to observe the early stage of domain creation phenomena, because it needs high spatial resolution, if one wants to see the domain directly. Raman spectroscopy provides us an opportunity to quantify the average domain size through a macroscopic measurement, because it is sensitive to the local structures such as boundary of domains. Thus we can obtain the information of domain creation and aggregation by observing time development of the Raman spectra during PIPT process.

Rubidium manganese hexacyanoferrate, $\operatorname{RbMn}\left[\mathrm{Fe}(\mathrm{CN})_{6}\right]$, is one of the well-studied materials among the family of Prussian blue analogues i.e., cyano-bridged metal complexes which show PIPT phenomena[6]. This compound shows charge transfer phase transition between high temperature (HT) state $\left(\mathrm{Fe}^{3+}-\mathrm{Mn}^{2+}\right)$ and low temperature (LT) state $\left(\mathrm{Fe}^{2+}-\mathrm{Mn}^{3+}\right)$ with large hysteresis loop around room temperature[7,8.9.10]. The coexistence of ferroelectricity and ferromagnetism in this complex is expected to exhibit various functionalities due to the interaction between electron and spin. [11]. The domain creation phenomena in the photo-induced phase transition between HT and LT state 
have been discussed by Fukaya et al.[12]. By using Raman spectroscopy, they have shown that the boundary (BO) between HT and LT domains increases in parallel with the LT state at the initial stage of the domain growth process. The irradiation time dependences of HT, LT and boundary components are explained qualitatively in terms of the model assuming the random charge transfer between Fe and $\mathrm{Mn}$ ions. Later, modified bimodal reaction model explained the initial region of the domain creation process more precisely [13]. However, the excitation power dependence and temporal development in a large time region have been still unclear. In this paper, we investigate generation dynamics of photo-induced domain in the prussian blue analogue $\operatorname{RbMnFe}(\mathrm{CN})_{6}$ compound by Raman spectroscopy. We succeeded in observing the domain growth dynamics including aggregation process and its excitation power dependence. At high excitation intensity, we found increase of the boundary component, indicating generation of small domains at early stage. We propose the theoretical model for charge transfer phase transition phenomena with the pair valence states of the adjacent $\mathrm{Fe}$ and $\mathrm{Mn}$ pairs and this model explains temporal development of boundary of HT and LT states qualitatively.

\section{Experimental}

The sample for optical measurements consisted of microcrystals with a typical size of a few micrometers[T1]. The sample was dispersed in liquid paraffin and sandwiched between two sapphire plates. Although we refer to as $\operatorname{RbMn}\left[\mathrm{Fe}(\mathrm{CN})_{6}\right]$ in this paper, the material used contained some amount of water and had a formula $\mathrm{Rb}_{0.58} \mathrm{Mn}\left[\mathrm{Fe}(\mathrm{CN})_{6}\right]_{0.78} \cdot 2.7 \mathrm{H}_{2} \mathrm{O}$. This sample is in HT state and does not show thermal phase transition phenomena from $4 \mathrm{~K}$ to RT. The Raman measurements were carried out under excitation by He-Cd laser (CW: wavelength; $442 \mathrm{~nm}$ ), using a triple grating monochromator equipped with a cooled CCD camera. Probe laser for Raman spectroscopy induces photo-induced phase transition phenomena. The typical exposure times for taking one Raman spectrum are several to several tens of seconds.

\section{Results}

Figure 1 shows the Raman spectra of $\mathrm{Rb}_{0.58} \mathrm{Mn}\left[\mathrm{Fe}(\mathrm{CN})_{6}\right]_{0.78} \cdot 2.7 \mathrm{H}_{2} \mathrm{O}$ at room temperature. At the very early stage of irradiation, a strong peak at $2160 \mathrm{~cm}^{-1}$ due to HT state is seen. As the irradiation time elapses, the probing laser induces the phase transition from HT state to LT state, thus the Raman spectrum changes. The new peaks around $2090 \mathrm{~cm}^{-1}$ (LT), $2130 \mathrm{~cm}^{-1}$ (LT), and $2240 \mathrm{~cm}^{-1}$ (BO) appear on the photo-induced (PI) state. We estimate the temporal developments of the HT, LT, and BO states from the intensity change of $2160 \mathrm{~cm}^{-1}, 2130 \mathrm{~cm}^{-1}$, and $2240 \mathrm{~cm}^{-1}$ peaks, respectively.

Figure 2 (a) and (b) show temporal developments of HT $\left(\mathrm{Fe}^{3+}-\mathrm{Mn}^{2+}\right)$, LT $\left(\mathrm{Fe}^{2+}-\mathrm{Mn}^{3+}\right)$, and BO $\left(\mathrm{Fe}^{3+}-\mathrm{Mn}^{3+}\right)$ states under low power $\left(3.2 \mathrm{~W} / \mathrm{cm}^{2}\right)$ and high power $\left(22 \mathrm{~W} / \mathrm{cm}^{2}\right)$ excitation, respectively. The vertical axes are the intensity of Raman peaks normalized by that of HT state at first 
measurement, during which the phase is assumed to be pure HT state. We can estimate fraction of the states by division of normalized peak intensity by the Raman cross section. The horizontal axes are number of the photons irradiated on the sample. As shown in Fig 2 (a) and (b), the Raman intensity corresponding to HT state decreases and that corresponding to LT state increases. This indicates the PIPT from HT state to PILT state. This behavior is qualitatively in agreement with the report by Fukaya et al.[12].

In Figure 2 (b), by increasing the excitation intensity, the Raman intensity of the BO peak increases abruptly and reaches to 0.7 . This indicates increase of the boundary component, suggesting creation of many small LT domains at early stage of phase transition phenomena. After reaching the maximum fraction, the BO peak decreases slowly and the LT peak increases. This suggests LT domain growth through aggregation process. As shown in Figure 2 (a), under the low power excitation, the Raman intensity of BO state increases slowly and reaches to only 0.15 . This suggests that, under the low power excitation, small numbers of nuclei appear and grow up. The ratio of BO peak to LT peak is smaller than that in high excitation case. This indicates the average sizes of domain are larger than that in latter case.

\section{Discussion}

It has been shown that the Slichter-Drickamer model reproduces the thermal phase transition phenomena between $\mathrm{HT}$ and LT states in $\mathrm{RbMnFe}(\mathrm{CN})_{6}$ compound [10]. However this model cannot describe dynamics of the boundary between HT and LT domains, because the amount of the boundary between HT and LT domains is not treated in this framework. We propose to extend the model to include the role of the boundary. Since the charge transfer occurs between two metal ions, the valence states of two adjacent metal ions play an important role in phase transition phenomena. In the $\mathrm{RbMnFe}(\mathrm{CN})_{6}$ compound, the pair valence states of the adjacent $\mathrm{Fe}$ and $\mathrm{Mn}$ pairs such as $\mathrm{Fe}^{3+}-\mathrm{Mn}^{2+}(\mathrm{HT}), \mathrm{Fe}^{2+}-\mathrm{Mn}^{3+}(\mathrm{LT})$, and $\mathrm{Fe}^{3+}-\mathrm{Mn}^{3+}$ (BO) can be taken as a basis for describing the charge transfer phase transition phenomena. However, as shown in Fig 3 (a), if the charge transfer occurs in the ion pair $\mathrm{Fe}^{3+}-\mathrm{Mn}^{2+}$ or $\mathrm{Fe}^{2+}-\mathrm{Mn}^{3+}$, the pair valence states of the neighboring Fe-Mn pairs are also changed. Therefore, it is necessary to consider the nearest-neighbor interactions for all the surrounding ion pairs. One electron transfer changes the pair valence states of (2Z-1) Fe-Mn pairs (Z: the coordination number of the metal site). We evaluate the contribution to the enthalpy from the nearest-neighbor ions by mean field approximations. This treatment is equivalent to the model which Hoo et al. suggested in spin crossover complex [14].

One electron transfer from $\mathrm{Mn}^{2+}$ to $\mathrm{Fe}^{3+}$ changes the fraction of the Fe-Mn pairs as follows;

$\Delta y_{32}^{+}=1-(Z-1) \frac{\gamma_{32}}{\gamma_{32}+\gamma_{33}}-(Z-1) \frac{\gamma_{32}}{\gamma_{32}+\gamma_{22}}$ 
$\Delta \gamma_{33}^{+}=(Z-1) \frac{\gamma_{32}}{\gamma_{32}+\gamma_{33}}-(Z-1) \frac{\gamma_{33}}{\gamma_{a 2}+\gamma_{33}}$

$\Delta y_{22}^{+}=(Z-1) \frac{\gamma_{32}}{\gamma_{32}+\gamma_{22}}-(Z-1) \frac{\gamma_{22}}{\gamma_{32}+\gamma_{22}}$

$\Delta \gamma_{23}^{+}=-1+(Z-1) \frac{\gamma_{22}}{\gamma_{32}+\gamma_{22}}+(Z-1) \frac{\gamma_{33}}{\gamma_{32}+\gamma_{33}}$

Here, $\gamma_{i j}$ are the fractions of the $\mathrm{Fe}^{\mathrm{i}+}-\mathrm{Mn}^{\mathrm{j}+}$ pairs and $\Delta \gamma^{+}{ }_{i j}$ are the change of the fraction of the $\mathrm{Fe}^{\mathrm{i}+}-\mathrm{Mn}^{\mathrm{j}+}$ pairs by one electron transfer from $\mathrm{Mn}^{2+}$ to $\mathrm{Fe}^{3+}$. The fraction change by charge transfer from LT state to HT state can be written likewise as follows;

$\Delta y_{32}^{-}=-1+(Z-1) \frac{\gamma_{33}}{\gamma_{32}+\gamma_{33}}+(Z-1) \frac{\gamma_{22}}{\gamma_{32}+\gamma_{22}}$

$\Delta \gamma_{33}=(Z-1) \frac{\gamma_{23}}{\gamma_{23}+\gamma_{33}}-(Z-1) \frac{\gamma_{33}}{\gamma_{23}+\gamma_{33}}$

$\Delta y_{22}^{-}=(Z-1) \frac{\gamma_{23}}{\gamma_{23}+\gamma_{22}}-(Z-1) \frac{\gamma_{22}}{\gamma_{23}+\gamma_{22}}$

$\Delta \gamma_{23}^{-}=1-(Z-1) \frac{\gamma_{23}}{\gamma_{23}+\gamma_{22}}-(Z-1) \frac{\gamma_{23}}{\gamma_{23}+\gamma_{33}}$

where $\Delta \gamma_{i j}^{-}$is change of fraction of the $\mathrm{Fe}^{\mathrm{i}+}-\mathrm{Mn}^{\mathrm{j}+}$ pairs by one electron transfer from $\mathrm{Fe}^{2+}$ to $\mathrm{Mn}^{3+}$. Figure3 (b) shows the potential energy between HT and LT state. This model is commonly used to discuss the simple phase transition between two states. $E_{\text {rel }}$ is the potential barrier between HT and LT states from average energy of HT and LT states, and $2 \Delta$ is the energy difference between HT and LT states. Transition probabilities of HT to LT state $\left(\mathrm{P}_{\mathrm{HL}}\right)$ and LT to HT state $\left(\mathrm{P}_{\mathrm{LH}}\right)$ are written as follows;

$$
\begin{aligned}
& P_{H L}=I_{0} \sigma \gamma_{32}+k^{+} \gamma_{32}, P_{L H}=k^{-} \gamma_{23} \\
& k^{+}=-k_{0} \exp \left(-\frac{E_{\text {rel }}+\left(\Delta-J a_{H T}-T \Delta S\right)}{k_{E} T}\right) \\
& k^{-}=-k_{0} \exp \left(-\frac{E_{\text {rel }}-\left(\Delta+J a_{L T}-T \Delta S\right)}{k_{E} T}\right)
\end{aligned}
$$

where $k_{0}$ is the reaction rate, $\Delta S$ is the entropy difference between HT and LT state, and $k_{B}$ is the Boltzmann constant, $I_{O}$ is the power of the excitation laser, and $\sigma$ is the coefficient describing photo-induced charge transfer efficiency. $a_{H T}$ and $a_{L T}$ are the number of the boundary states $\left(\mathrm{Fe}^{2+}-\mathrm{Mn}^{2+}\right.$ and $\left.\mathrm{Fe}^{3+}-\mathrm{Mn}^{3+}\right)$ in the nearest neighbor of $\mathrm{Fe}^{3+}-\mathrm{Mn}^{2+}$ and $\mathrm{Fe}^{2+}$ and $\mathrm{Mn}^{3+}$ pairs, respectively. $J$ expresses cooperative interactions due to the irregular combination of the valences on the boundary: If the valence states of the ions are changed due to a charge transfer transition in the case that its nearest neighbor ion is in the boundary state then energy of HT and LT states increase by $J$ and these states become unstable as shown in Fig3 (b). We can derive rate equations for $\gamma_{i j}$ from 
equations (3).

$\frac{d \gamma_{i j}}{d t}=\left(I_{0} \sigma+k^{+}\right) \gamma_{32} \Delta \gamma_{i j}^{+}-k^{-} \gamma_{23} \Delta \gamma_{i j}^{-} \quad \mathrm{i}=2,3, \mathrm{j}=2,3$

, where $\mathrm{k}^{+}, \mathrm{k}^{-}, \gamma_{\mathrm{ij}}{ }^{+}, \gamma_{\mathrm{ij}}{ }^{-}$are defined by Eq. (4), (5), (1), (2), respectively. Figure 4 shows the temporal development of the fractions of $\mathrm{Fe}^{3+}-\mathrm{Mn}^{2+}, \mathrm{Fe}^{2+}-\mathrm{Mn}^{3+}$, and $\mathrm{Fe}^{3+}-\mathrm{Mn}^{3+}$ calculated with this model for the parameters reported in the caption to Fig. 4. $\Delta$ and $\Delta S$ are estimated from the heat capacity measurements [9]. Fraction of $\mathrm{Fe}^{3+}-\mathrm{Mn}^{2+}$ decreases and $\mathrm{Fe}^{2+}-\mathrm{Mn}^{3+}$ increases with time, and $\mathrm{Fe}^{3+}-\mathrm{Mn}^{3+}$ increases abruptly and after reaching maximum relaxes slowly. These behaviors are qualitatively in agreement with the experimental result as shown in Fig2 (b). At the beginning, the $\mathrm{Fe}^{3+}-\mathrm{Mn}^{2+}$ decreases by $11, \mathrm{Fe}^{2+}-\mathrm{Mn}^{3+}$ increases by 1 , and $\mathrm{Fe}^{3+}-\mathrm{Mn}^{3+}$ increases by 10 with one charge transfer transition from $\mathrm{Mn}^{2+}$ to $\mathrm{Fe}^{3+}$. This corresponds to the first step of creating new LT nuclei in the HT domains. This region has been already discussed in previous report [12]. The fraction of $\mathrm{Fe}^{2+}-\mathrm{Mn}^{3+}$ increases with time, and when the fractions of $\mathrm{Fe}^{3+}-\mathrm{Mn}^{2+}$ and $\mathrm{Fe}^{3+}-\mathrm{Mn}^{3+}$ become equal, the fraction of $\mathrm{Fe}^{3+}-\mathrm{Mn}^{3+}$ does not change by charge transfer, because $\Delta \gamma_{33}{ }^{+}$is 0 (see Eq. 1). In the region where fraction of $\mathrm{Fe}^{3+}-\mathrm{Mn}^{3+}$ is larger than that of $\mathrm{Fe}^{3+}-\mathrm{Mn}^{2+}$, fraction of $\mathrm{Fe}^{3+}-\mathrm{Mn}^{3+}$ decrease by charge transfer. This corresponds to the aggregation process of domains. As shown in Eq. 3, the rate of photo-induced change is proportional to fraction of $\mathrm{Fe}^{3+}-\mathrm{Mn}^{2+}$, thus the decrease of $\mathrm{Fe}^{3+}-\mathrm{Mn}^{3+}$ slows down at large $t$.

With the low excitation density $I_{0} / 10$, the calculation shows that the maximum fraction of $\mathrm{Fe}^{3+}-\mathrm{Mn}^{3+}$ decreases. This behavior agrees with the experimental result. However, it has a peak in this simple assumption using the mean field approximation, while the experimental result does not have a peak as shown in Fig 2 (b). The mean flied theory is not appropriate to treat interaction between very dilute domains in the initial state of low excitation condition. To describe this case, more advanced treatment such as Monte Carlo simulation is needed.

\section{Conclusions}

We investigated the domain growth and aggregation dynamics in PIPT phenomena by Raman spectroscopy. We observed the abrupt increase and slow relaxation of the boundary. This suggests the small domain creation and growth phenomena in the early stage of PIPT and aggregation process in the next stage. These behaviors are explained qualitatively by the theoretical model using mean field approximation for evaluating the excess enthalpy due to irregular ion pairs at the boundary.

\section{Acknowledgements}

This work was supported in part by the Grant-in Aid for Scientific Researches from the Ministry of Education, Culture, Sports, Science, and Technology of Japan, the CREST program of JST and the NEXT project from JSPS. 


\section{Reference}

[1] S. Koshihara, Y. Tokura, K. Takeda, and T. Koda, Phys. Rev. Lett. 68 (1992) 1148.

[2] S. Koshihara, Y. Takahashi, H. Sakai, Y. Tokura, and T. Luty, J. Phys. Chem. B 103 (1999) 2592.

[3] Y. Ogawa, S. Koshihara, K. Koshino, T. Ogawa, C. Urano, and H. Takagi, Phys. Rev. Lett. 84 (2000) 3181.

[4] K. Nasu (ed.), Photoinduced phase transitions, World Scientific Publishing, Singapore, (2004).

[5] H. Watanabe, H. Hirori, G. Molnar, A. Bousseksou, and K. Tanaka, Phys. Rev. B, 79 (2009) 180405(R).

[6] H. Tokoro, K. Hashimoto, and S. Ohkoshi, J. Magn. Magn. Mater. 310 (2007) 1422.

[7] H. Tokoro, S. Ohkoshi, T. Matsuda, and K. Hashimoto, Inorg.Chem., 43 (2004) 5231.

[8] S. Ohkoshi, T. Matsuda, H. Tokoro, and K. Hashimoto, Chem. Mater., 17 (2005) 81.

[9] S. Ohkoshi, Hiroko Tokoro, and Kazuhito Hashimoto, Coord. Chem. Rev., 249 (2005) 1830.

[10] H. Tokoro, S. Miyashita, K. Hashimoto, and S. Ohkoshi, Phys. Rev. B 73 (2006) 172415.

[11] S. Ohkoshi, H.Tokoro, T.Matsuda, H. Takahashi, H. Irie, and K. Hashimoto, Angew. Chem. Int. Ed., 46 (2007) 3238.

[12] R. Fukaya, M. Nakajima, H. Tokoro, S. Ohkoshi, and T. Suemoto, J. Chem. Phys., 134 (2009) 154505.

[13] T. Suemoto, R. Fukaya, A. Asahara, M. Nakajima, T. Tokoro, and S.Ohkoshi, Phys. Stat. Sol. (b), 248, 2 (2011) 477.

[14] B. Hoo, K. Boukheddaden, and F. Varret, Eur. Phys.J. B, 17 (2000) 449. 


\section{Figure Captions}

Fig. 1. Raman spectra of $\mathrm{C}-\mathrm{N}$ stretching modes at room temperature. Under irradiation by probing laser $(442 \mathrm{~nm})$ the spectrum changes with time indicating PIPT from HT state to LT state. The Raman peaks at $2090 \mathrm{~cm}^{-1}, 2130 \mathrm{~cm}^{-1}, 2160 \mathrm{~cm}^{-1}$, and $2240 \mathrm{~cm}^{-1}$ are ascribed to $\mathrm{Fe}^{2+}-\mathrm{Mn}^{3+}(\mathrm{LT})$, $\mathrm{Fe}^{2+}-\mathrm{Mn}^{3+}(\mathrm{LT}), \mathrm{Fe}^{3+}-\mathrm{Mn}^{2+}(\mathrm{HT})$, and $\mathrm{Fe}^{3+}-\mathrm{Mn}^{3+}(\mathrm{BO})$, respectively.

Fig. 2. Temporal development of the Raman intensity of $\mathrm{Fe}^{3+}-\mathrm{Mn}^{2+}\left(2160 \mathrm{~cm}^{-1}\right), \mathrm{Fe}^{2+}-\mathrm{Mn}^{3+}(2130$ $\left.\mathrm{cm}^{-1}\right)$, and $\mathrm{Fe}^{3+}-\mathrm{Mn}^{3+}\left(2240 \mathrm{~cm}^{-1}\right)$ under the $3.2 \mathrm{~W} / \mathrm{cm}^{2}$ (a) or $22 \mathrm{~W} / \mathrm{cm}^{2}($ b) irradiation.

Fig. 3. (a) Schematic figure of the charge transfer between Fe and Mn ions. The phase transition from HT to LT state is caused by the electron transfer from $\mathrm{Mn}^{2+}$ to $\mathrm{Fe}^{3+}$. (b) Schematic figure of the potential energy between HT and LT state. The enthalpy difference between HT and LT state is $2 \Delta$, and there is a potential barrier $E_{\text {rel }}$ between HT and LT state.

Fig. 4. Temporal development of the fraction of $\mathrm{Fe}^{2+}-\mathrm{Mn}^{3+}, \mathrm{Fe}^{3+}-\mathrm{Mn}^{2+}$, and $\mathrm{Fe}^{3+}-\mathrm{Mn}^{3+}$ under the irradiation obtained from numerical solutions. The parameters used are $I_{0}=0.05, K_{0}=0.5, E_{\text {rel }}=1500$ $\mathrm{K}, \Delta=1.7 \mathrm{kJmol}^{-1}, \Delta S=6 \mathrm{JK}^{-1} \mathrm{~mol}^{-1}, J=500 \mathrm{~K}, T=300 \mathrm{~K}$, and $Z=6(3 \mathrm{D})$. 
Fig.1

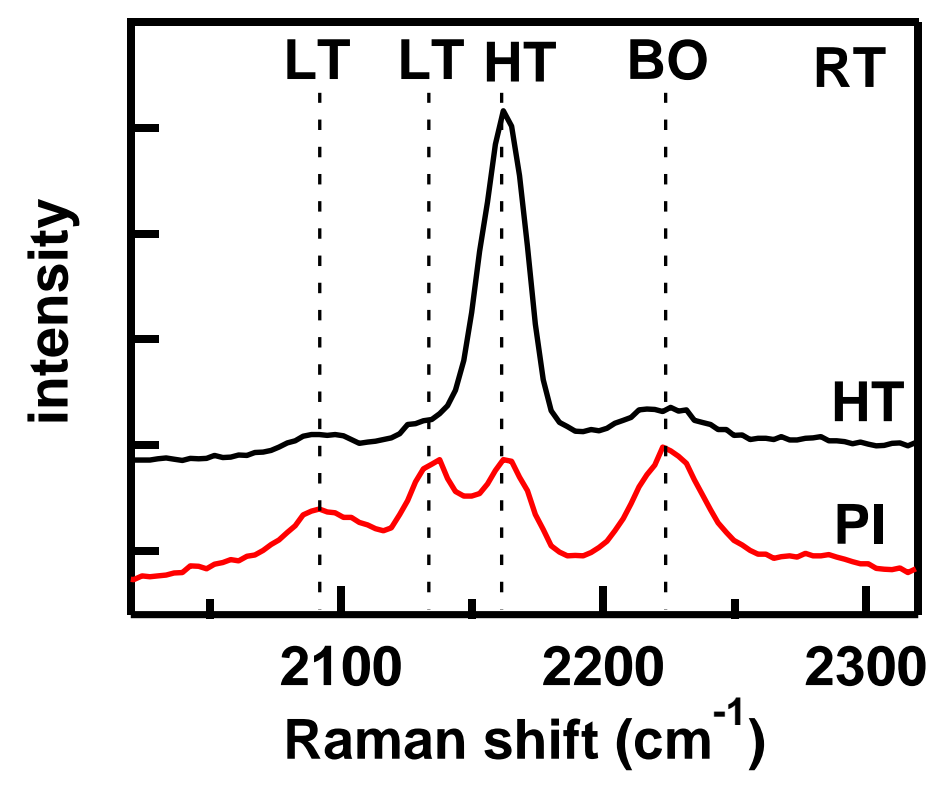

H.Watanabe 
Fig.2

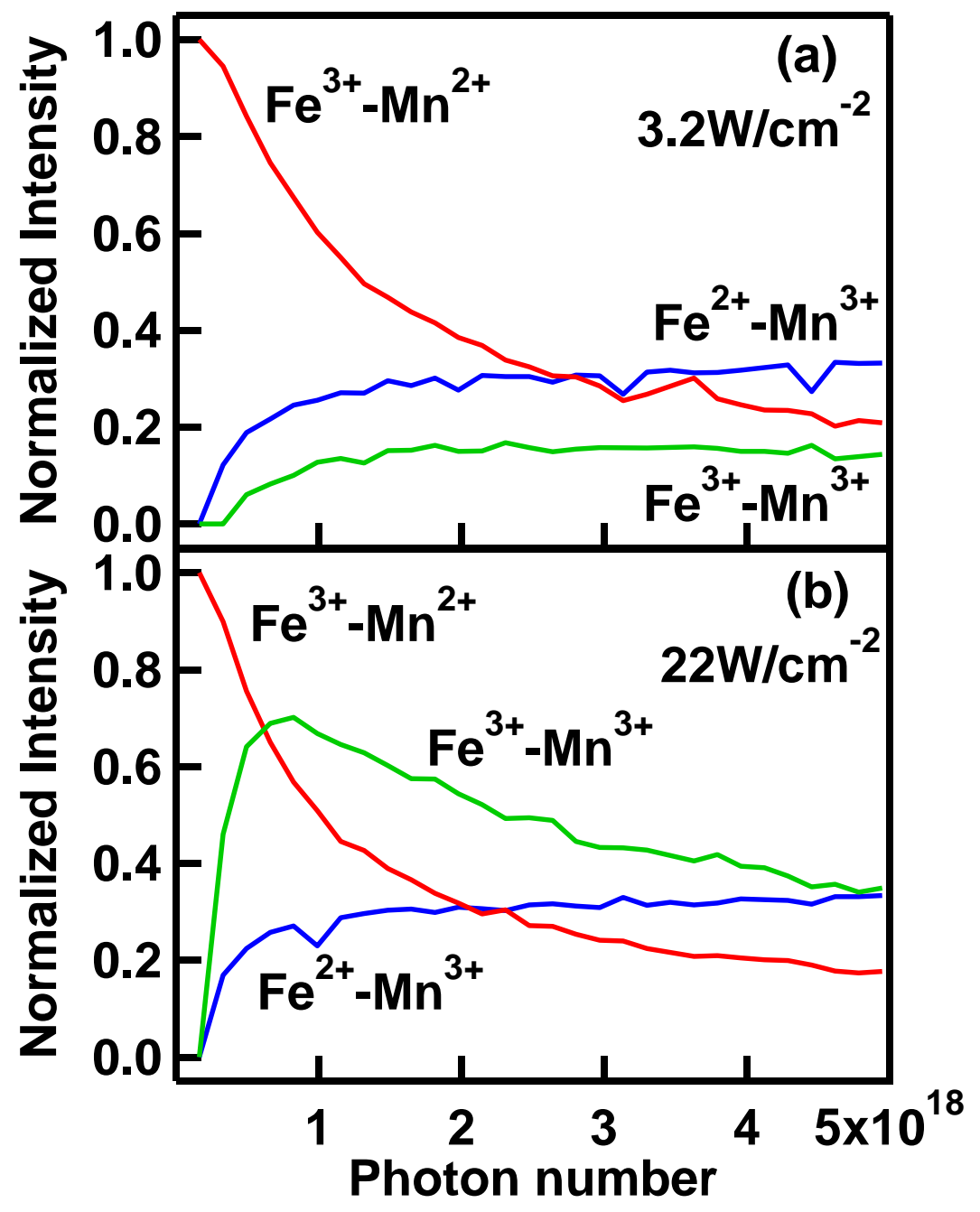

H.Watanabe 
Fig.3

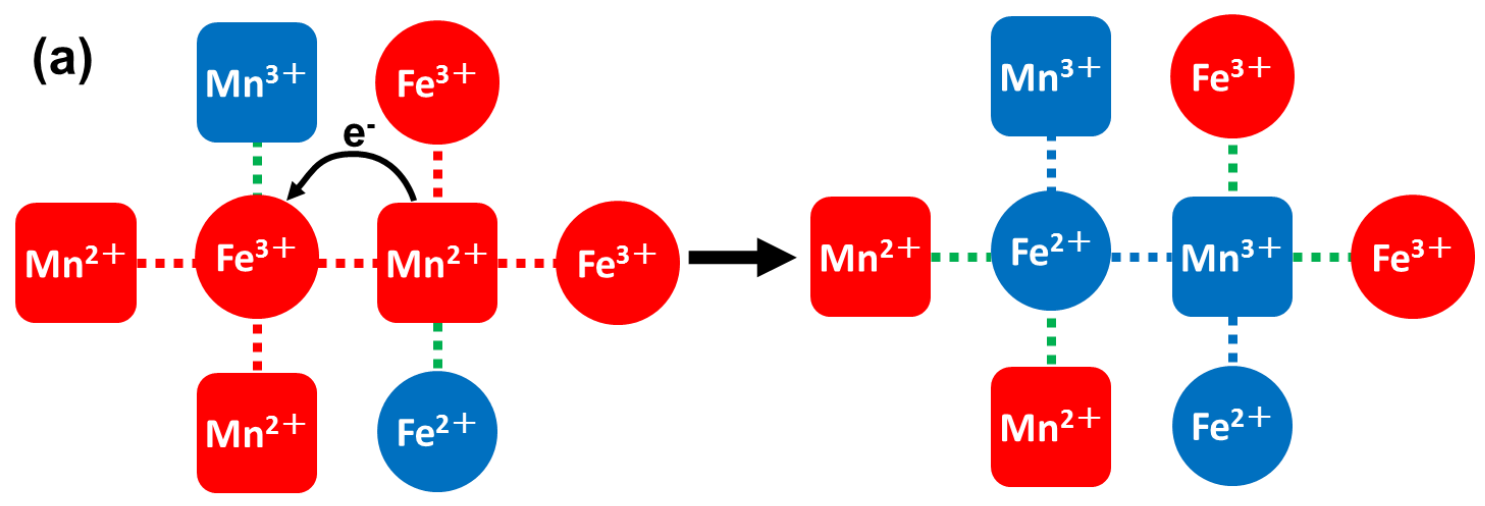

(b)

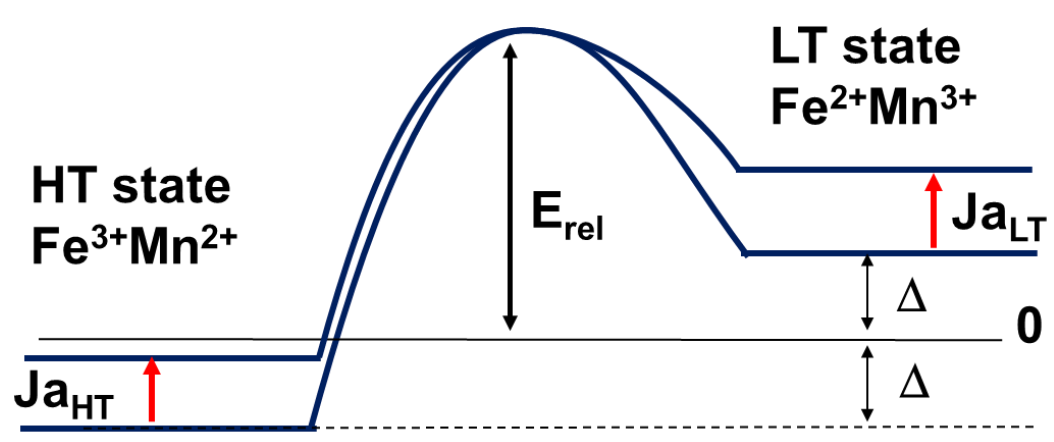

H.Watanabe 
Fig.4

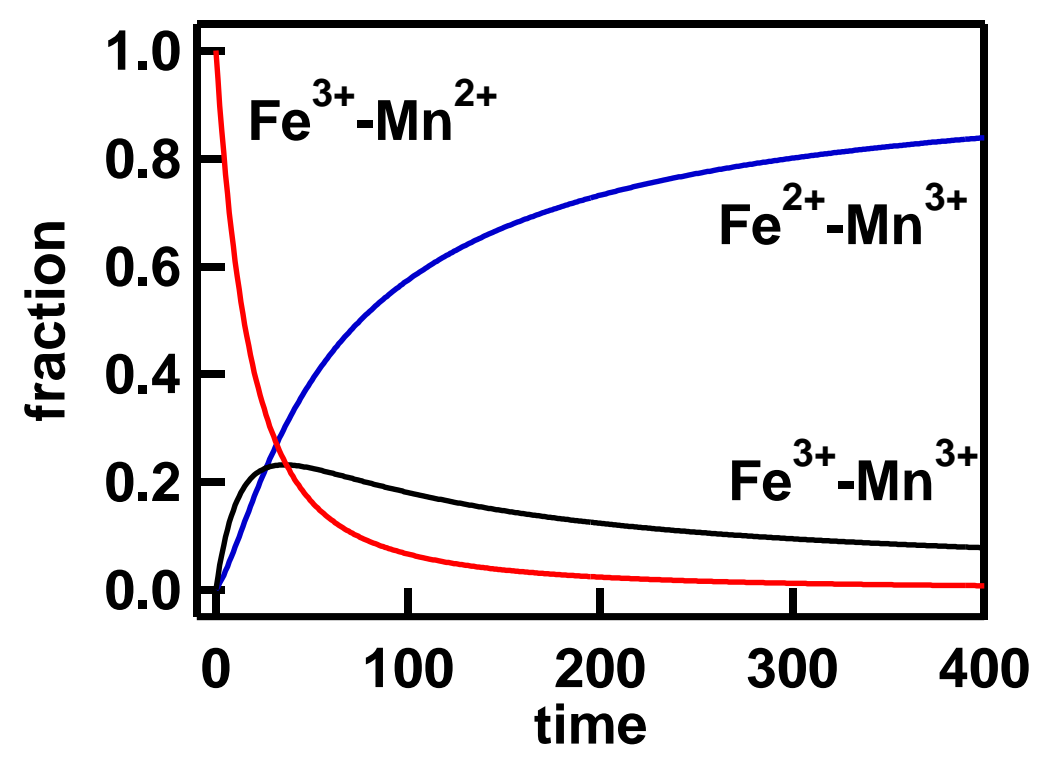

H.Watanabe 